\title{
PHYSIOTHERAPY IN A SPECIALISED HAND UNIT
}

\section{SUE HOLT, M.C.S.P.*}

During the year 1976. over one thousand new hand cases and three thousand repeat cases were seen at the

* Senior Physiotherapist, Groote Schuur Hospital, Cape Town.
Hand Unit of Groote Schuur Hospital. The majority of these patients either passed through the Physiotherapy Department or that of Occupational Therapv. Close teamwork is an essential factor in this field. This article aims at describing the treatment given by 
the Physiotherapy Department. The classification of cases from a physiotherapeutic point of view is as follows:-

\section{Traum:}

Fractures. dislocations. crush injurics. Finger tip injuries. traumatic amputations. Cut tendons. Nerve lesions.

\section{Infections}

\section{Miscellaneous}

Carpal tunnel syndrome, ganglia. Dupuytren's contracturc. benign giant cell tumours and cysts.

\section{Systemic}

Rheumatoid arthritis. systemic lupus erythematosus and gout

\section{Comgenital}

\section{TRAUMA}

Fractures, dislocations, crush injuries

The general principle of treatment for all fracrures early mobilisation with immediate reduction of oedema. Splintage is uscd minimally. Fractures of the phalanges are strapped to the adjacent finger. A five $\mathrm{cm}$ crêpe bandage for metacarpal fractures is sufficient, care being taken to ensure that the metacarpal joints are frec for mobilisation. In the event of an unstable fracture a Kirschner wire may be inserted and if this involves the joint the mobilisation must bc left until later. However, for the majority of fractures and crush injuries. the first aim is to reduce oedema as quickly as possible. as it may lead to adhesions. poor blood supply and loss of functional mobility.

The Hot Box, through which warm air is circulated. has been specially designed so that the patient can sit with the hand and arm elevated. (See Fig. 1). In this position as much range of active movement as possible is encouraged. This is progressed to a squeezing and pumping action on a roll of sorbo rubber. In cases with severe oedema a patient can spend up to four hours working in the Hot Box, both in the Departments of Physiotherapy and Occupational Therapy. It has been found that if excrcises are started as soon as possible. oedema is not a great problem. If swelling has been present for over 48 hours it may become consolidated and minimise the final range of movement. As soon as oedema is reduced. Functional activity is stressed by active and gentle passive movements. After three weeks all fixation is removed and the majority of patients gain full range of movement within six to eight weeks.

Dislocations of the joints present greater problems and must be immobilised for approximately two to three weeks. Due to the damage to the capsule and surrounding structures, the joints are often stiff and it is extrcmely difficult to regain full range. Again. active and passive exerciscs are given and ultrasound in water may be used although its efficacy is open to question. Ice baths have also been found to help in increasing range. (Wynn Parry. C. B.. 1973).

Tratumatic ampurations and more particularly finger tip injuries in children are fairly common in the Western Cape when fingers get caught in doors due 10 the strong South Easterly wind. Treatment is the same. to reduce oedema and regain full functional activity as soon as possible.

Cur rendons present a surgical challenge. Briefly they are treatcd by primary suture/repair, secondary suture/ repair. tendon grafts or insertion of silastic rods prior to tendon grafting.

Il primary repair is done. the hand is immobilised in plaster of Paris for three weeks. During this time the only treatment necessary is 10 teach and supervise shoulder elevation exercises to prevent oedema. After three weeks. the plaster is removed and active mobilisation is commenced.

For a secondary repair, the skin is merely closed after the injury and the tendons will be repaired at a later stage. In this instance it is essential that the physiotherapist gains and maintains FULL passive range of all joints and encourages the patient to keep the hand and \& fingers as supple as possible prior to the second operation. Immobilisation is again three weeks and active movement can then be startcd

One must distinguish between cut extensor tendons and cut flexor tendons. The re-education and mobilisa-

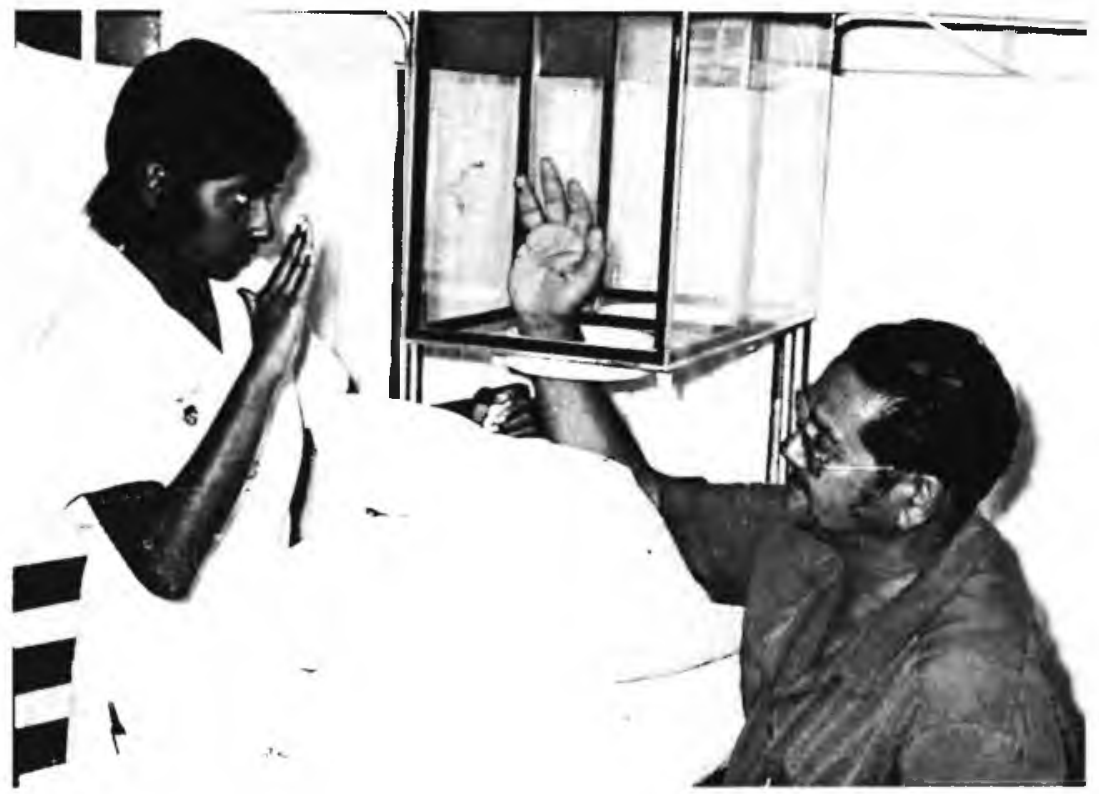

Fig. 1. 
tion following an extensor tendon repair is straightforward. The patient strengthens up quickly, although a $5^{\circ}$ to $10^{\circ}$ extensor tendon lag may persist.

The re-education following a flexor tendon repair is, however, much slower and more arduous. Following immobilisation, i.e. from the fourth week ACTIVE flexion and extension, and during the fifth week gentle passive flexion is allowed. From the sixth week, passive stretching into extension is started and dynamic splintage worn at night in order to increase the range of extension. It must be stressed that FLEXION is the functional movement of the hand and it is more important to regain the maximum possible range of flexion at the expense of full extension. This stage of the tendon repair is vital and the patient should be prepared to spend practically the entire day in the Physiotherapy and Occupational Therapy Departments. Scarring and adhesions may be forming and these must be kept to a minimum. Passive range of flexion MUST be obtained and maintained. Active movement should be encouraged continuously with the use of sponge grips.

Besides the conventional method of heat, our Department has also found ice baths to be effective in gaining an increase in range both passive and active. An explanation for this is uncertain, but it may be due to the increased vasodilatation of the vessels in the hand following vasoconstriction. (Wynn Parry, C. B., 1973).

Ultrasound and scar massage are important in reducing tendon adherence. The effects of ultrasound have never been conclusively proved, but it has a part to play providing dosages of $1-2$ Watts $/ \mathrm{cm}^{2}$, are used (Wynn Parry, C. B., 1973). This is applied either directly to the area or through the medium of water. It should be used in conjunction with scar massage. During this stage active movement is VITALLY important. If only one or two fingers are involved, it is of benefit to strap these to the "good fingers" to help with flexion. In the later stages of re-education resisted exercises can be given, such as proprioceptive neuromuscular facilitation techniques or manual labour in the workshops. Throughout the re-education process, measurements should always be taken and if the result is a finger which can finally flex to within $1 \mathrm{~cm}$ of palmar crease, it can be considered successful.

Tendon grafts are usually determined by the level of the lesion. For example, in "No-mans-land" suturing would produce excessive scarring and hence no effective pull through of the tendons. The rehabilitation after a graft follows the same pattern as for a direct repair.

In cases where severe scarring is already present, silastic rods may be inserted so that a sheath can form prior to grafting. This is usually done in approximately three or four months. With this type of patient, full passive movements must be maintained whilst the rods are in situ.

Nerve lesions are repaired by primary repair, secondary repair or nerve grafts. The physiotherapy treatment is principally the same as for tendon lesions. Immediate post-operative instruction to reduce oedema is given. If it is a nerve cut at the wrist that is repaired, then active movement of the fingers can begin immediately as far as the immobilising plaster will allow. If tendons and nerves are involved, a secondary nerve repair is usually done by the time active mobilisation of the tendons has already started. Rehabilitation of the hand follows the same pattern, except that the immobilisation period is six weeks. Commonly, either the median and/or ulnar nerve are involved and reeducation should also involve returning sensation. This is usually carried out by occupational therapy. If, however, the radial nerve has been severed, this is sutured and the patient wears a lively splint until the nerve grows down to the extensor muscles. If this faik
tendon transfers may be done. tendon transfers may be done. Thereafter, re-education
of wrist, finger and thumb extension would be necessar,

\section{INFECTIONS}

These represent a large percentage of hand seen. The basic principles of treatment hand cass drainage and excision of any slough; no antihiotision, given unless essential; frequent dressings anplics ari duction of oedema and movement are encoured; reshould be stressed that the rules regarding dressing. It very strictly kept to in the Hand Unit. Dry dress are the minimum of bandaging and specially dressings, stockinette gloves and finger stalls to prevent angned striction from tight bandaging, strapping or tube $\mathrm{re}$ are applied. The Hot Box is used with the gaus hand in elevation to reduce swelling, passive and ments are given and active movement is encoure. When the wound is closed, it may be necessaged. soak the hand in warm water mixed with a to Savlon. This helps to remove all the dead tissue and the skin is then massaged with lanolin cream. Riteither from animals, insects or humans fall int Bitcategory and the treatment follows the same principles,

\section{MISCELANEOUS}

Patients with ganglia, carpal tunnel syndrome, Dupuytren's contracture, trigger finger, cysts, etc, are seen immediately post-operatively by the Physiotherapy Department in order to give instructions to prevent oedema and lack of mobility. The patient is instructed in the importance of doing elevation exercises of the hand and shoulder (a minimum of 200 times per day) and to move the unaffected areas, such as metacarpal and interphalangeal joints. As a routine, the patient returns to the Department the following day for a check and this should be all the treatment required. However. with a release of a Dupuytren's contracture, further passive stretching and treatment may be required, at the specific instruction of the surgeon.

\section{SYSTEMIC CONDITIONS AND CONGENITAL HAND DEFORMITIES}

These are not common in this Unit and should be treated according to individual need. Splintage may be the predominant issue in which case the Occupational Therapy Department will be primarily responsible.

\section{CONCLUSION}

Two vital points emerge. Firstly, the patient, that THE HAND, should be treated as soon as possib. before complications have arisen. Secondly, the importance of teamwork cannot be stressed sufficiently enough. It is no use working merely as a physiotherapist in the isolation of the Department. One must be present at the clinics as a member of the team, consisting of the surgeons, sister, occupational therapist and physiotherapist, as well as the social worker who is involved in placing the patient with an injured hand in suitable employment.

\section{Acknowledgements}

My thanks for the help in compiling this article are due to Dr. Cecil Bloch and Dr. Martin Singer, surgeons attached to the Hand Unit of Groote Schuur Hospital, to Mr. B. G. Moth, Chief Physiotherapist of the Physiotherapy Department and to Dr. H. Reeve-Sanders, Medical Superintendent, Groote Schuur Hospital, for permission to publish the article.

\section{References:}

Wynn Parry, C. B. (1973) Chapter 6-Principles of Rehabilitation in Rehabilitation of the Hand. Butterworths, London 3rd ed. pp. 243 - 270. 